\title{
A Novel Hybrid Function Projective Synchronization between Different Fractional-Order Chaotic Systems
}

\author{
Ping Zhou ${ }^{1,2}$ and Xiao-You Yang ${ }^{2}$ \\ ${ }^{1}$ Center of System Theory and Its Applications, Chongqing University of Posts and Telecommunications, \\ Chongqing 400065, China \\ ${ }^{2}$ Key Laboratory of Network Control and Intelligent Instrument of Ministry of Education, \\ Chongqing University of Posts and Telecommunications, Chongqing 400065, China
}

Correspondence should be addressed to Ping Zhou, zhouping@cqupt.edu.cn

Received 5 April 2011; Accepted 9 June 2011

Academic Editor: Antonia Vecchio

Copyright (C) 2011 P. Zhou and X.-Y. Yang. This is an open access article distributed under the Creative Commons Attribution License, which permits unrestricted use, distribution, and reproduction in any medium, provided the original work is properly cited.

\begin{abstract}
An adaptive hybrid function projective synchronization (AHFPS) scheme between different fractional-order chaotic systems with uncertain system parameter is addressed in this paper. In this proposed scheme, the drive and response system could be synchronized up to a vector function factor. This proposed scheme is different with the function projective synchronization (FPS) scheme, in which the drive and response system could be synchronized up to a scaling function factor. The adaptive controller and the parameter update law are gained. Two examples are presented to demonstrate the effectiveness of the proposed scheme.
\end{abstract}

\section{Introduction}

In nonlinear science, chaos synchronization is a hot topic, which has attracted much attention from scientists and engineers in the last few years [1-13]. Projective synchronization (PS) first reported by Mainieri and Rehacek [14] has been extensively investigated in recent years because it can obtain faster communication. Projective synchronization (PS) is characterized that the drive and response system could be synchronized up to a scaling factor. This proportional feature can be used to extend binary digital to $M$-nary digital communication [14] for getting faster communication. Recently, a new type of projective synchronization method [15-17], called function projective synchronization (FPS), is put forward. The drive and response system could be synchronized up to a scaling function factor in function projective synchronization (FPS). FPS could be used to get more security in application 
to secure communications, because the unpredictability of the scaling function in FPS can additionally enhance the security of communication.

At present, the FPS mentioned so far involved mainly the integer-order chaotic systems, and the parameters are exactly known in advance. But in many practical situations, many fractional-order systems yet exhibit chaotic behavior. The parameters of these fractional-order systems in social science and biological science cannot be known entirely. To the best of our knowledge, there are few results about the FPS for fractional-order chaotic systems with uncertain system parameter and there are few results about the FPS for a vector function factor. Motivated by the above discussion, an adaptive hybrid function projective synchronization (AHFPS) scheme between different fractional-order chaotic systems with uncertain system parameters is investigated in this paper. The drive and response system could be synchronized up to a vector function factor in this proposed scheme. This technique is applied to achieve the AHFPS between different fractional-order Lorenz systems with one uncertain system parameter, and the AHFPS between the fractionalorder Lorenz system with one uncertain system parameter and the fractional-order Chen system. The numerical simulations demonstrate the validity and feasibility of the proposed method.

The organization of this paper is as follows. In Section 2, the definition of the AHFPS is given and the AHFPS scheme between different fractional-order chaotic systems with uncertain system parameter is presented. In Section 3, two examples are used to verify the effectiveness of the proposed scheme. The conclusion is finally drawn in Section 4.

\section{The Fractional Derivatives and AHFPS Scheme}

The Caputo definition of the fractional derivative, which is sometimes called smooth fractional derivative, is described as

$$
D^{q} f(t)=\frac{1}{\Gamma(m-q)} \int_{0}^{t} \frac{f^{(m)}(\tau)}{(t-\tau)^{q+1-m}} d \tau, \quad m-1<q<m,
$$

where $D^{q}$ denotes the Caputo definition of the fractional derivative, $m$ is the smallest integer larger than $q, f^{(m)}(t)$ is the $m$-order derivative in the usual sense, $\Gamma(\cdot)$ is the gamma function.

The fractional-order chaotic drive and response systems can be described as follows, respectively:

$$
\begin{gathered}
D^{q_{d}} x=D(x), \\
D^{q_{r}} z=R(z, \beta),
\end{gathered}
$$

where $q_{d}$ and $q_{r}$ are fractional orders satisfying $0<q_{d}<1,0<q_{r}<1$, and $q_{r}$ may be different with $q_{d} . x \in R^{n}$ and $z \in R^{n}$ are the state vectors of the drive system (2.2) and response system (2.3), respectively. $\beta$ is the system parameter. $D, R: R^{n} \rightarrow R^{n}$ may be different continuous nonlinear vector functions. 
If parameter $\beta$ in system (2.3) is unknown, a parameter update law is designed, and a controller $\psi$ is added to the original system (2.3), we obtain the controlled response system (2.4) with parameter update laws (2.5)

$$
\begin{gathered}
D^{q_{r}} z=R(z, \tilde{\beta})+\psi, \\
D^{q_{r}} \tilde{\beta}=p(x, z, \tilde{\beta}),
\end{gathered}
$$

where $\tilde{\beta}$ is unknown parameter. Controller $\psi$ is an $n \times 1$ real matrix to be designed, and $p(x, z, \widetilde{\beta})$ is a real scalar function to be designed.

Definition 2.1. For the drive system (2.2) and controlled response system (2.4) with parameter update laws (2.5), it is said to be adaptive hybrid function projective synchronization (AHFPS) if there exist a controller $\psi$ and a real scalar function $p(x, z, \widetilde{\beta})$ such that

$$
\lim _{t \rightarrow+\infty}\|e\|=\lim _{t \rightarrow+\infty}\|z-K(x) x\|=0, \quad \lim _{t \rightarrow+\infty}\left\|e_{\beta}\right\|=\lim _{t \rightarrow+\infty}\left\|\tilde{\beta}-\beta_{0}\right\|=0,
$$

where $\|\cdot\|$ is the Euclidean norm and $\beta_{0}$ is the "true" value of the "unknown" parameter $\tilde{\beta} . K(x)$ is an $n \times n$ real matrix, and matrix elements $k_{i j}(x)(i, j=1,2, \ldots, n)$ are continuous bounded functions. $e_{i}=z_{i}-\sum_{j=1}^{n} k_{i j} x_{j}$ and $e_{\beta}=\tilde{\beta}-\beta_{0}$ are called the AHFPS error.

Remark 2.2. If $K(x)=k I$ and $k \in R$ is a constant, then the AHFPS problem will be reduced to adaptive projective synchronization (APS), where $I$ is an $n \times n$ identity matrix. If $K(x)=\operatorname{diag}\left(k_{1}, k_{2}, \ldots, k_{n}\right)$ and $k_{i} \in R$ are constant, then the adaptive modified projective synchronization (AMPS) will appear. And if $K(x)=K$, and $K$ is a constant matrix, then the adaptive hybrid projective synchronization (AHPS) will appear. If $K(x)=\operatorname{diag}\left(k_{1}(x)\right.$, $\left.k_{2}(x), \ldots, k_{n}(x)\right)$ and $k_{i}(x)(i=1,2, \ldots n)$ are continuous bounded functions, then the adaptive function projective synchronization (AFPS) will appear, that is, the AFPS is also the special case of the proposed scheme.

Remark 2.3. Based on the idea of tracking control, in order to achieve the goal of $\lim _{t \rightarrow+\infty}\|e\|=$ $\lim _{t \rightarrow+\infty}\|z-K(x) x\|=0$, we can let $K(x) x$ be a reference signal. Then, AHFPS between fractional-order chaotic system (2.2) and fractional-order chaotic system (2.4) belongs to the problem of tracking control, that is, the output signal $z$ in system (2.4) follows the reference signal $K(x) x$ ultimately.

In the next, we will discuss how to choose a controller $\psi$ and a parameter update laws.

First, the "true" value of the "unknown" parameter $\tilde{\beta}$ is chosen as $\beta_{0}$, and we define a compensation controller $\psi_{1}(x) \in R^{n}$ for response system (2.4),

$$
\psi_{1}(x)=D^{q_{r}}(K(x) x)-R\left(K(x) x, \beta_{0}\right),
$$

and choose controller $\psi$ as

$$
\psi=\psi_{1}(x)+\psi_{2}
$$

where $\psi_{2}$ is an $n \times 1$ vector function which will be designed later. 
According to the controller (2.8) and the compensation controller (2.7), the response system (2.4) can be depicted as

$$
D^{q_{r}} z=R(z, \tilde{\beta})+D^{q_{r}}(K(x) x)-R\left(K(x) x, \beta_{0}\right)+\psi_{2} .
$$

Using $e=y-K(x) x$, one has

$$
D^{q_{r}} e=R(z, \tilde{\beta})-R\left(K(x) x, \beta_{0}\right)+\psi_{2}
$$

In generally, we can get

$$
R(z, \tilde{\beta})-R\left(K(x) x, \beta_{0}\right)=\xi_{1}\left(x, z, \beta_{0}\right)\left(\begin{array}{c}
e \\
e_{\beta}
\end{array}\right) .
$$

where $\xi_{1}\left(x, z, \beta_{0}\right)$ is an $n \times(n+1)$ real matrix and $\left(\begin{array}{c}e \\ e_{\beta}\end{array}\right)=\left(e_{1}, e_{2}, \ldots, e_{n}, e_{\beta}\right)^{T}$ is an $(n+1) \times 1$ real matrix.

Second, we define vector function $\psi_{2}$ as

$$
\psi_{2}=\xi_{2}\left(x, z, \beta_{0}\right)\left(\begin{array}{c}
e \\
e_{\beta}
\end{array}\right),
$$

where $\xi_{2}\left(x, z, \beta_{0}\right)$ is an $n \times(n+1)$ real matrix to be designed.

From (2.10), (2.11), and (2.12), we have

$$
D^{q_{r}} e=\left[\xi_{1}\left(x, z, \beta_{0}\right)+\xi_{2}\left(x, z, \beta_{0}\right)\right]\left(\begin{array}{c}
e \\
e_{\beta}
\end{array}\right) .
$$

Finally, let the parameter update law be

$$
D^{q_{r}} \tilde{\beta}=p(x, z, \tilde{\beta})=\tau\left(\begin{array}{c}
e \\
e_{\beta}
\end{array}\right),
$$

where $\tau$ is an $1 \times(n+1)$ real matrix to be designed later. Because the Caputo derivative of a constant is zero, (2.14) can be rewritten as

$$
D^{q_{r}}\left(\tilde{\beta}-\beta_{0}\right)=D^{q_{r}} e_{\beta}=\tau\left(\begin{array}{c}
e \\
e_{\beta}
\end{array}\right) .
$$

According to (2.13) and (2.15), we have

$$
\left(\begin{array}{c}
D^{q_{r}} e \\
D^{q_{r}} e_{\beta}
\end{array}\right)=\left(\begin{array}{c}
\xi_{1}\left(x, z, \beta_{0}\right)+\xi_{2}\left(x, z, \beta_{0}\right) \\
\tau
\end{array}\right)\left(\begin{array}{c}
e \\
e_{\beta}
\end{array}\right),
$$

where $\left(\underset{\tau}{\xi_{1}\left(x, z, \beta_{0}\right)+\xi_{2}\left(x, z, \beta_{0}\right)}\right)$ is an $(n+1) \times(n+1)$ real matrix. 
By (2.16), we know that the AHFPS between fractional-order system (2.2) with controlled response fractional-order system (2.4) and the uncertain parameter $\tilde{\beta}$ could be identified, and transformed into the following problem: choose suitable $n \times(n+1)$ real matrix $\xi_{2}\left(x, z, \beta_{0}\right)$ and $1 \times(n+1)$ real matrix $\tau$ such that system (2.16) is asymptotically convergent to zero.

Remark 2.4. According to the above, $K(x) x$ may be an arbitrary given reference signal, therefore the AHFPS between fractional-order chaotic systems and integer-order chaotic systems belongs to this class of problems if we choose the reference signal $K(x) x$ as being the output of one of the integer-order chaotic systems.

Theorem 2.5. If real matrix $\xi_{2}\left(x, z, \beta_{0}\right)$ and $\tau$ in system (2.16) are selected such that

$$
P\left(\begin{array}{c}
\xi_{1}\left(x, z, \beta_{0}\right)+\xi_{2}\left(x, z, \beta_{0}\right) \\
\tau
\end{array}\right)+\left(\begin{array}{c}
\xi_{1}\left(x, z, \beta_{0}\right)+\xi_{2}\left(x, z, \beta_{0}\right) \\
\tau
\end{array}\right) P=-Q
$$

where $P$ is a real symmetric positive definite matrix, $Q$ is a real symmetric positive semidefinite matrix, and $H$ stands for conjugate transpose of a matrix, then

$$
\lim _{t \rightarrow+\infty}\|e\|=\lim _{t \rightarrow+\infty}\|z-K(x) x\|=0, \quad \lim _{t \rightarrow+\infty}\left\|e_{\beta}\right\|=\lim _{t \rightarrow+\infty}\left\|\tilde{\beta}-\beta_{0}\right\|=0
$$

Proof. Assume that $\lambda$ is one of the eigenvalues of matrix $\left(\underset{\xi_{1}}{\left(x, z, \beta_{0}\right)+\xi_{2}\left(x, z, \beta_{0}\right)}\right)$ and the corresponding nonzero eigenvector is $\Psi$, that is,

$$
\left(\begin{array}{c}
\xi_{1}\left(x, z, \beta_{0}\right)+\xi_{2}\left(x, z, \beta_{0}\right) \\
\tau
\end{array}\right) \Psi=\lambda \Psi .
$$

Multiplying the above equation left by $\Psi^{H} P$, we get

$$
\Psi^{H} P\left(\left(\begin{array}{c}
\xi_{1}\left(x, z, \beta_{0}\right)+\xi_{2}\left(x, z, \beta_{0}\right) \\
\tau
\end{array}\right) \Psi\right)=\Psi^{H} P(\lambda \Psi)
$$

By a similar argument, we also can obtain

$$
\left(\Psi^{H}\left(\begin{array}{c}
\xi_{1}\left(x, z, \beta_{0}\right)+\xi_{2}\left(x, z, \beta_{0}\right) \\
\tau
\end{array}\right)\right) P \Psi=\left(\bar{\lambda} \Psi^{H}\right) P \Psi
$$

According to (2.20) and (2.21), we can obtain

$$
\lambda+\bar{\lambda}=\frac{\left\{\Psi^{H}\left[P\left(\xi_{1}\left(x, z, \beta_{0}\right)+\xi_{2}\left(x, z, \beta_{0}\right)\right)+\left(\underset{\tau}{\xi_{1}\left(x, z, \beta_{0}\right)+\xi_{2}\left(x, z, \beta_{0}\right)}\right)^{H} P\right] \Psi\right\}}{\Psi^{H} P \Psi} .
$$


Since $P\left(\dot{\xi}_{1}\left(x, z, \beta_{0}\right)+\grave{\xi}_{2}\left(x, z, \beta_{0}\right)\right)+\left(\underset{\xi_{1}}{\grave{1}_{1}\left(x, z, \beta_{0}\right)+\dot{\xi}_{2}\left(x, z, \beta_{0}\right)}\right)^{H} P=-Q$ and $P, Q$ are real symmetric positive definite matrix and real symmetric positive semidefinite matrix, respectively, then

$$
\begin{gathered}
\Psi^{H} Q \Psi \geq 0, \quad \Psi^{H} P \Psi>0 \\
\lambda+\bar{\lambda}=-\frac{\Psi^{H} Q \Psi}{\Psi^{H} P \Psi} \leq 0
\end{gathered}
$$

So, we can obtain

$$
|\arg \lambda| \geq \frac{\pi}{2}>\frac{q_{r} \pi}{2}
$$

According to the stability theory of fractional-order systems [18], the equilibrium point in (2.16) is asymptotically stable.

Therefore,

$$
\lim _{t \rightarrow+\infty}\|e\|=\lim _{t \rightarrow+\infty}\|z-K(x) x\|=0, \quad \lim _{t \rightarrow+\infty}\left\|e_{\beta}\right\|=\lim _{t \rightarrow+\infty}\left\|\tilde{\beta}-\beta_{0}\right\|=0
$$

The proof is completed.

This theorem indicates that system (2.16) can asymptotically converge to zero. It implies that the AHFPS between drive system (2.2) and controlled response system (2.4) with uncertain parameter $\tilde{\beta}$ will be obtained.

\section{Applications}

In this section, to illustrate the effectiveness of the proposed synchronization scheme, the AHFPS between different fractional-order Lorenz systems with one uncertain system parameter and the AHFPS between the fractional-order Lorenz system with one uncertain system parameter and the fractional-order Chen system are considered and the numerical simulations are performed.

First, we introduce the numerical solution of fractional differential equations in [19]. All the numerical simulation of fractional-order system in this paper is based on [19]. Consider the following fractional-order system:

$$
\begin{aligned}
& \frac{d^{q_{1}} x}{d t^{q_{1}}}=f(x, y), \quad 0<q_{1}, q_{2}<1, \\
& \frac{d^{q_{2}} y}{d t^{q_{2}}}=g(x, y),
\end{aligned}
$$


with initial condition $\left(x_{0}, y_{0}\right)$. Now, set $h=T / N, t_{n}=n h(n=0,1,2 \ldots, N)$. The previous system can be discretized as follows:

$$
\begin{aligned}
& x_{n+1}=x_{0}+\frac{h^{q_{1}}}{\Gamma\left(q_{1}+2\right)}\left[f\left(x_{n+1}^{p}, y_{n+1}^{p}\right)+\sum_{j=0}^{n} \alpha_{1, j, n+1} f\left(x_{j}, y_{j}\right)\right] \\
& y_{n+1}=y_{0}+\frac{h^{q_{2}}}{\Gamma\left(q_{2}+2\right)}\left[g\left(x_{n+1}^{p}, y_{n+1}^{p}\right)+\sum_{j=0}^{n} \alpha_{2, j, n+1} g\left(x_{j}, y_{j}\right)\right]
\end{aligned}
$$

where

$$
\begin{aligned}
& x_{n+1}^{p}=x_{0}+\frac{1}{\Gamma\left(q_{1}\right)} \sum_{j=0}^{n} b_{1, j, n+1} f\left(x_{j}, y_{j}\right), \\
& y_{n+1}^{p}=y_{0}+\frac{1}{\Gamma\left(q_{2}\right)} \sum_{j=0}^{n} b_{2, j, n+1} g\left(x_{j}, y_{j}\right),
\end{aligned}
$$

and, for $i=1,2$,

$$
\begin{aligned}
& \alpha_{i, j, n+1}= \begin{cases}n^{q_{i}+1}-\left(n-q_{i}\right)(n+1)^{q_{i},} & j=0, \\
(n-j+2)^{q_{i}+1}+(n-j)^{q_{i}+1}-2(n-j+1)^{q_{i}+1}, & 1 \leq j \leq n, \\
1, & j=n+1,\end{cases} \\
& b_{i, j, n+1}=\frac{h^{q_{i}}}{q_{i}}\left[(n-j+1)^{q_{i}}-(n-j)^{q_{i}}\right], \quad 0 \leq j \leq n .
\end{aligned}
$$

The error of this approximation is described as follows:

$$
\left|x\left(t_{n}\right)-x_{n}\right|=o\left(h^{p_{1}}\right), \quad p_{1}=\min \left(2,1+q_{1}\right),\left|y\left(t_{n}\right)-y_{n}\right|=o\left(h^{p_{2}}\right), \quad p_{2}=\min \left(2,1+q_{2}\right) .
$$

\subsection{The AHFPS between the Fractional-Order Lorenz Chaotic System with Different Fractional Order}

The famous Lorenz system [20], the first chaotic attractor model in a 3D autonomous system, is described as follows:

$$
\begin{gathered}
D^{q_{r}} z_{1}=\sigma\left(z_{2}-z_{1}\right), \\
D^{q_{r}} z_{2}=\beta z_{1}-z_{1} z_{3}-z_{2}, \\
D^{q_{r}} z_{2}=z_{1} z_{2}-\gamma z_{3} .
\end{gathered}
$$




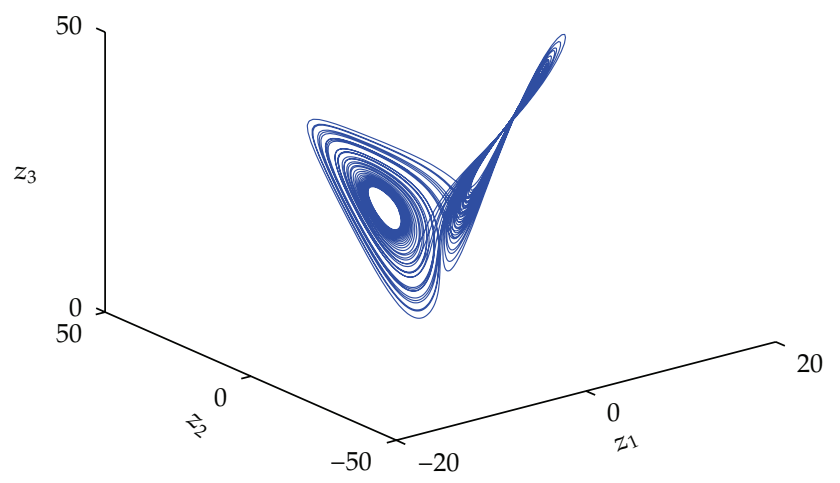

Figure 1: Chaotic attractors of the fractional-order Lorenz system (3.6) for $q_{r}=0.998$.

where system parameters $(\sigma, \beta, \gamma)=(10,28,8 / 3)$. I. Grigorenko and E. Grigorenko [20] pointed out that fractional-order Lorenz system (3.6) exhibits chaotic behavior for fractional order $q_{r} \geq 0.993$. The chaotic attractor for $q_{r}=0.998$ is shown in Figure 1.

If fractional-order $q$ in fractional-order Lorenz system is 0.995 , we can rewrite the fractional-order Lorenz system as

$$
\begin{gathered}
D^{q_{d}} x_{1}=\sigma\left(x_{2}-x_{1}\right), \\
D^{q_{d}} x_{2}=\beta x_{1}-x_{1} x_{3}-x_{2}, \\
D^{q_{d}} x_{2}=x_{1} x_{2}-\gamma x_{3},
\end{gathered}
$$

where $q_{d}=0.995$. Now, let the fractional-order Lorenz system (3.7) be drive system and parameter $\beta$ unknown in fractional-order Lorenz system (3.6). The fractional-order system (3.6) with uncertain parameters $\tilde{\beta}$ is described by

$$
\begin{gathered}
D^{q_{r}} z_{1}=10\left(z_{2}-z_{1}\right), \\
D^{q_{r}} z_{2}=\tilde{\beta} z_{1}-z_{1} z_{3}-z_{2}, \\
D^{q_{r}} z_{3}=z_{1} z_{2}-\frac{8 z_{3}}{3} .
\end{gathered}
$$

According to the above, we can get the controlled response system (3.9) with uncertain parameter $\tilde{\beta}$, and parameter update laws (3.10):

$$
\begin{gathered}
\left(\begin{array}{c}
D^{q_{r}} z_{1} \\
D^{q_{r}} z_{2} \\
D^{q_{r}} z_{3}
\end{array}\right)=\left(\begin{array}{c}
10\left(z_{2}-z_{1}\right) \\
\tilde{\beta} z_{1}-z_{1} z_{3}-z_{2} \\
z_{1} z_{2}-\frac{8 z_{3}}{3}
\end{array}\right)+\psi, \\
D^{q_{r}} \tilde{\beta}=p(x, z, \tilde{\beta})=\tau\left(\begin{array}{c}
e \\
e_{\beta}
\end{array}\right) .
\end{gathered}
$$


Discrete Dynamics in Nature and Society

According to the above, we can obtain

$$
\xi_{1}\left(x, z, \beta_{0}\right)=\left(\begin{array}{cccc}
-10 & 10 & 0 & 0 \\
\beta_{0}-z_{3} & -1 & -\sum_{j=1}^{3} k_{1 j}(x) x_{j} & z_{1} \\
z_{2} & \sum_{j=1}^{3} k_{1 j}(x) x_{j} & -8 / 3 & 0
\end{array}\right) .
$$

Now, the parameter update laws and real matrix $\xi_{2}\left(x, z, \beta_{0}\right)$ are chosen as

$$
D^{q_{r}} \tilde{\beta}=p(x, z, \tilde{\beta})=\tau\left(\begin{array}{c}
e \\
e_{\beta}
\end{array}\right)=-z_{1} e_{2}, \quad \xi_{2}\left(x, z, \beta_{0}\right)=\left(\begin{array}{cccc}
0 & z_{3}-\beta_{0}-10 & -z_{2} & 0 \\
0 & 0 & 0 & 0 \\
0 & 0 & 0 & 0
\end{array}\right) .
$$

Therefore,

$$
\left(\begin{array}{c}
\xi_{1}\left(x, z, \beta_{0}\right)+\xi_{2}\left(x, z, \beta_{0}\right) \\
\tau
\end{array}\right)=\left(\begin{array}{cccc}
-10 & z_{3}-\beta_{0} & -z_{2} & 0 \\
\beta_{0}-z_{3} & -1 & -\sum_{j=1}^{3} k_{1 j}(x) x_{j} & z_{1} \\
z_{2} & \sum_{j=1}^{3} k_{1 j}(x) x_{j} & -\frac{8}{3} & 0 \\
0 & -z_{1} & 0 & 0
\end{array}\right) .
$$

Choosing real symmetric positive definite matrix $P=\operatorname{diag}(1,1,1,1)$, we can get

$$
P\left(\begin{array}{c}
\xi_{1}\left(x, z, \beta_{0}\right)+\xi_{2}\left(x, z, \beta_{0}\right) \\
\tau
\end{array}\right)+\left(\begin{array}{c}
\xi_{1}\left(x, z, \beta_{0}\right)+\xi_{2}\left(x, z, \beta_{0}\right) \\
\tau
\end{array}\right) P=\operatorname{diag}\left(-20,-2,-\frac{16}{3}, 0\right) .
$$

Choosing real symmetric positive semidefinite matrix $Q=\operatorname{diag}(20,2,16 / 3,0)$, we can obtain

$$
P\left(\begin{array}{c}
\xi_{1}\left(x, z, \beta_{0}\right)+\xi_{2}\left(x, z, \beta_{0}\right) \\
\tau
\end{array}\right)+\left(\begin{array}{c}
\xi_{1}\left(x, z, \beta_{0}\right)+\xi_{2}\left(x, z, \beta_{0}\right) \\
\tau
\end{array}\right) P=-Q
$$


So, the AHFPS between fractional-order Lorenz system (3.7) and controlled response system (3.9) with uncertain parameters $\tilde{\beta}$ can be achieved. For example, choose

$$
K(x)=\left(\begin{array}{ccc}
x_{1}+x_{3} & 0.5 & 1 \\
2 & 1+0.1 x_{1} x_{2} & -1 \\
-0.5 & -2 & 1+x_{2}
\end{array}\right) .
$$

The corresponding numerical result is shown in Figure 2, in which the initial conditions are $x(0)=(0,1,2)^{T}, z(0)=(5.5,0,4)^{T}, \widetilde{\beta}(0)=31$, and ep $=e_{\beta}$ and the "true" value of the "unknown" parameter is chosen as $\beta_{0}=28$, respectively.

\subsection{The AHFPS between the Fractional-Order Lorenz Chaotic System and the Fractional Order Chen Chaotic System}

Chen and Ueta introduced another chaotic system, called Chen chaotic system, which is similar but not topologically equivalent to the Lorenz system. Chen chaotic system [21] is given by

$$
\begin{gathered}
\frac{d x_{1}}{d t}=a\left(x_{2}-x_{1}\right), \\
\frac{d x_{2}}{d t}=(c-a) x_{1}-x_{1} x_{3}+c x_{2}, \\
\frac{d x_{3}}{d t}=x_{1} x_{2}-b x_{3}
\end{gathered}
$$

where $(a, b, c)=(35,3,28)$. Its corresponding fractional-order system is described as follows,

$$
\begin{gathered}
D^{q_{d}} x_{1}=a\left(x_{2}-x_{1}\right), \\
D^{q_{d}} x_{2}=(c-a) x_{1}-x_{1} x_{3}+c x_{2}, \\
D^{q_{d}} x_{3}=x_{1} x_{2}-b x_{3},
\end{gathered}
$$

Tavazoei and Haeri [22] pointed out that fractional-order Chen system exhibits chaotic behavior for fractional order $q_{d} \geq 0.83$. When $q_{d}=0.95$, the chaotic attractor is shown in Figure 3.

Now, let the fractional-order Chen system (3.18) be drive system and fractional-order Lorenz system (3.6) with unknown parameter $\beta$ as response system. According to the above, 


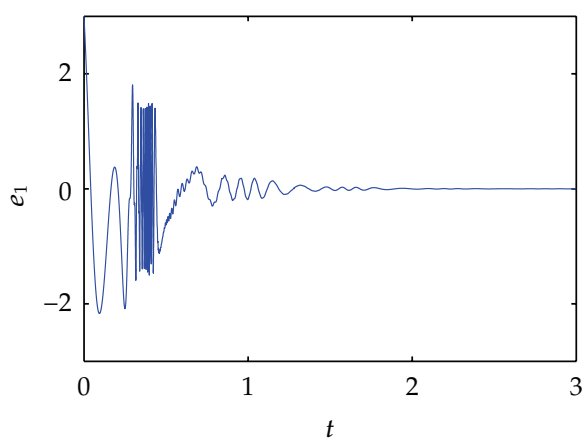

(a)

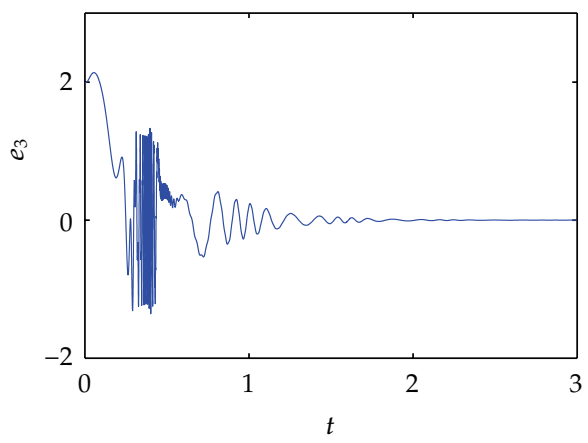

(c)

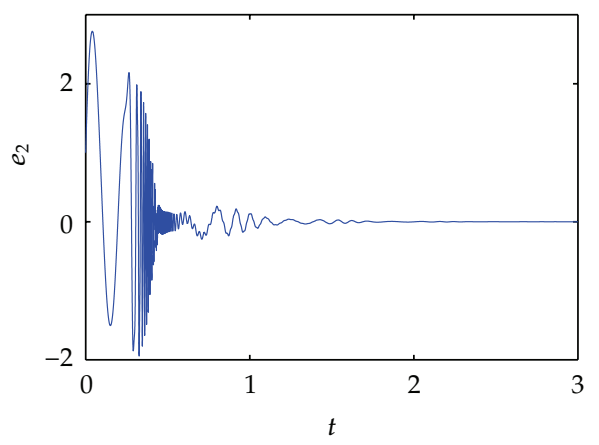

(b)

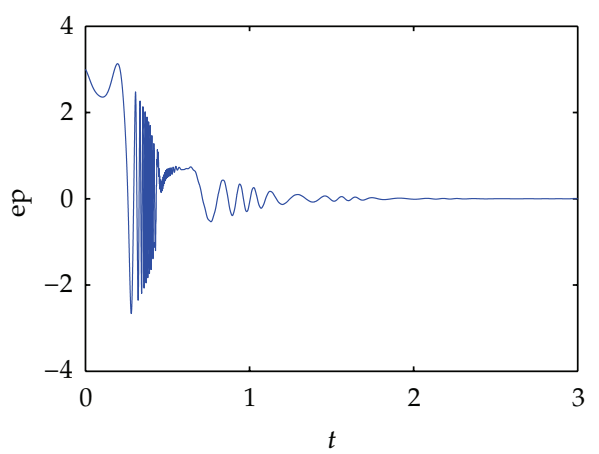

(d)

Figure 2: Time evolution of the AHFPS error.

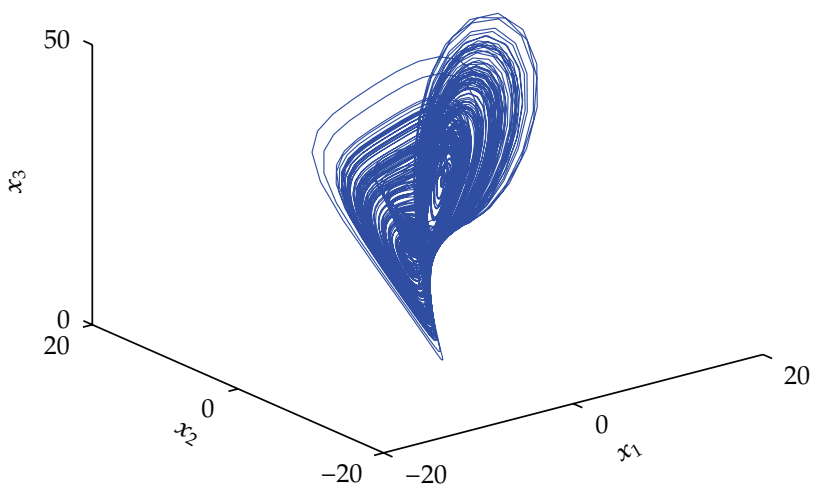

Figure 3: Chaotic attractors of the fractional-order Chen system (3.18) for $q_{d}=0.95$. 
we can get the controlled response system with uncertain parameter $\tilde{\beta}(3.9)$, and parameter update laws (3.10). Similar to the above, we can obtain

$$
\xi_{1}\left(x, z, \beta_{0}\right)=\left(\begin{array}{cccc}
-10 & 10 & 0 & 0 \\
\beta_{0}-z_{3} & -1 & -\sum_{j=1}^{3} k_{1 j}(x) x_{j} & z_{1} \\
z_{2} & \sum_{j=1}^{3} k_{1 j}(x) x_{j} & -\frac{8}{3} & 0
\end{array}\right) .
$$

The parameter update laws and real matrix $\xi_{2}\left(x, z, \beta_{0}\right)$ are chosen as

$$
D^{q_{r}} \tilde{\beta}=-z_{1} e_{2}, \quad \xi_{2}\left(x, z, \beta_{0}\right)=\left(\begin{array}{cccc}
0 & z_{3}-\beta_{0}-10 & -z_{2} & 0 \\
0 & 0 & 0 & 0 \\
0 & 0 & 0 & 0
\end{array}\right) .
$$

If we choose real symmetric positive definite matrix $P=\operatorname{diag}(1,1,1,1)$ and real symmetric positive semidefinite matrix $Q=\operatorname{diag}(20,2,16 / 3,0)$, we can get

$$
P\left(\begin{array}{c}
\xi_{1}\left(x, z, \beta_{0}\right)+\xi_{2}\left(x, z, \beta_{0}\right) \\
\tau
\end{array}\right)+\left(\begin{array}{c}
\xi_{1}\left(x, z, \beta_{0}\right)+\xi_{2}\left(x, z, \beta_{0}\right) \\
\tau
\end{array}\right) P=-Q .
$$

So, the AHFPS between the fractional-order Chen system (3.18) and controlled response system (3.9) with uncertain parameters $\tilde{\beta}$ can be achieved. For example, choose

$$
K(x)=\left(\begin{array}{ccc}
1+0.1 x_{1} x_{2} & 0.5 & 1 \\
2 & x_{1}+x_{3} & -1 \\
-0.5 & -2 & x_{2}+x_{1}
\end{array}\right)
$$

The corresponding numerical result is shown in Figure 4, in which the initial conditions are $x(0)=(0,1,2), z(0)=(5.5,1,2)$, and $\tilde{\beta}(0)=43$, and the "true" value of the "unknown" parameter is chosen as $\beta_{0}=40$, respectively. The chaotic attractor of the fractional-order Lorenz system (3.6) with $(\sigma, \alpha, \beta)=(10,40,8 / 3)$ for $q_{r}=0.998$ is shown in Figure 5.

According to the numerical results in Figures 2 and 4, we can obtain that the errors are indeed close to zero. This means that the adaptive hybrid function projective synchronization (AHFPS) between different chaotic systems can be achieved finally.

\section{Conclusion}

In this paper, an adaptive hybrid function projective synchronization (AHFPS) scheme between different fractional-order chaotic systems with uncertain system parameter is addressed. The drive and response system could be synchronized up to a vector function factor 


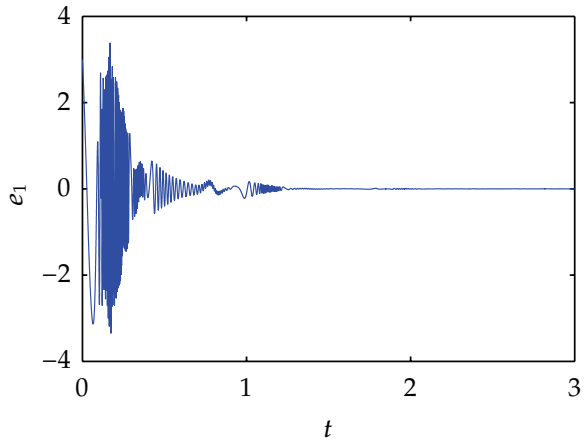

(a)

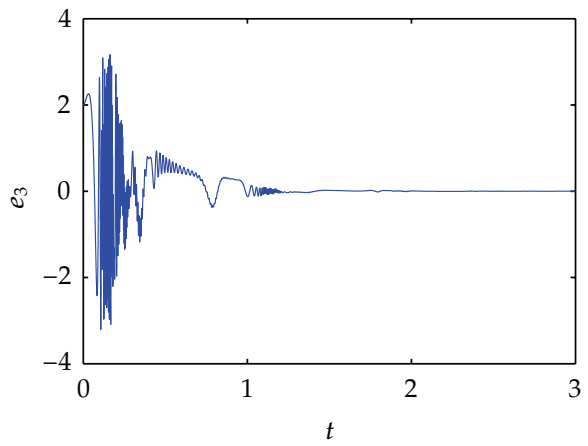

(c)

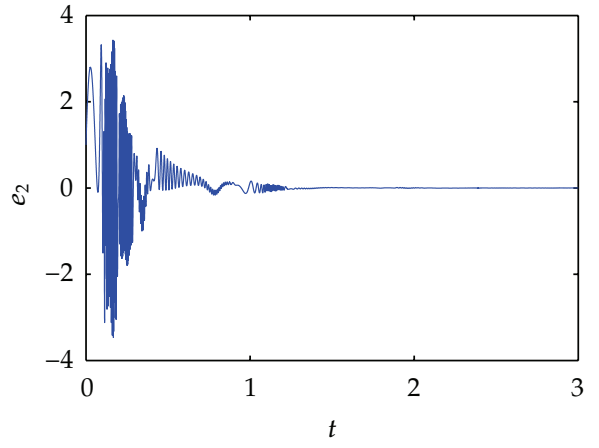

(b)

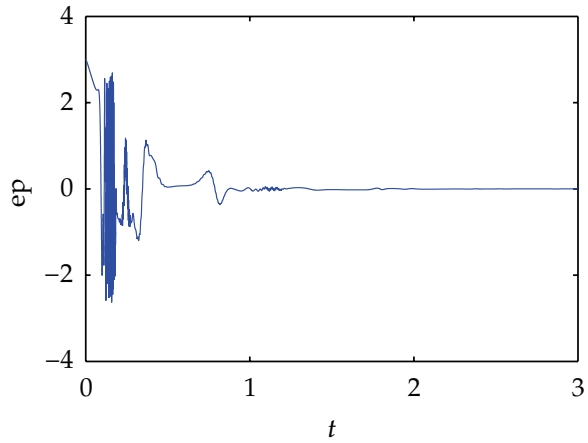

(d)

Figure 4: Time evolution of the AHFPS error.

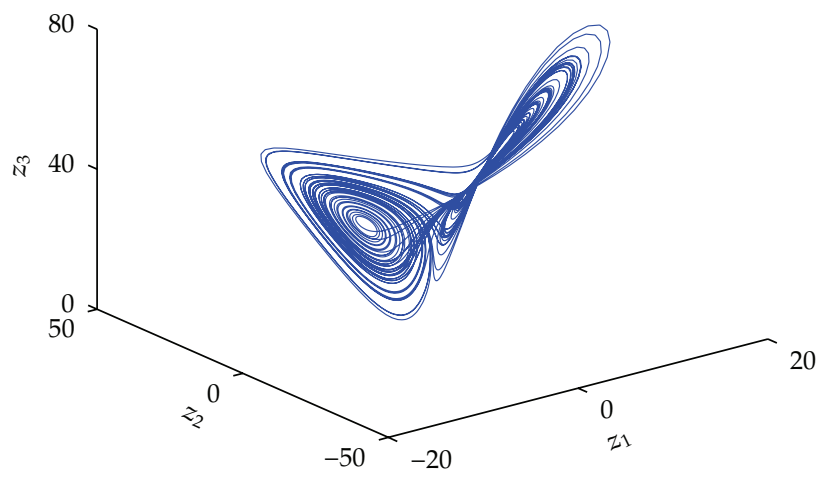

Figure 5: Chaotic attractors of the fractional-order Lorenz system $(3.6)$ with $(\sigma, \alpha, \beta)=(10,40,8 / 3)$ for $q_{r}=0.998$.

in this proposed scheme. This is different with the function projective synchronization (FPS) scheme, in which the drive and response system could be synchronized up to a scaling function factor. Based on the stability theory of fractional-order system, an adaptive controller and the parameter update law are obtained. The AHFPS between different fractionalorder Lorenz chaotic system with uncertain system parameter and the AHFPS between 
the fractional-order Lorenz chaotic systems with uncertain system parameter and the fractional-order Chen chaotic system are discussed. The numerical simulations demonstrate the validity and feasibility of the proposed scheme.

\section{Acknowledgments}

The paper is supported jointly by Foundation of Science and Technology project of Chongqing Education Commission under Grant KJ110525, National Natural Science Foundation of China under Grant 61004042, and Natural Science Foundation Project of CQ CSTC $2009 B$ B2417.

\section{References}

[1] L. M. Pecora and T. L. Carroll, "Synchronization in chaotic systems," Physical Review Letters, vol. 64, no. 8, pp. 821-824, 1990.

[2] E. Ott, C. Grebogi, and J. A. Yorke, "Controlling chaos," Physical Review Letters, vol. 64, no. 11, pp. 1196-1199, 1990.

[3] G. Chen and X. Dong, From Chaos to Order: Methodologies, Perspectives and Applications, vol. 24 of World Scientific Series on Nonlinear Science. Series A: Monographs and Treatises, World Scientific, River Edge, NJ, USA, 1998.

[4] H. Salarieh and A. Alasty, "Adaptive synchronization of two chaotic systems with stochastic unknown parameters," Communications in Nonlinear Science and Numerical Simulation, vol. 14, no. 2, pp. 508-519, 2009.

[5] X.-Y. Wang and J.-M. Song, "Synchronization of the fractional order hyperchaos Lorenz systems with activation feedback control," Communications in Nonlinear Science and Numerical Simulation, vol. 14, no. 8, pp. 3351-3357, 2009.

[6] H. Du, Q. Zeng, C. Wang, and M. Ling, "Function projective synchronization in coupled chaotic systems," Nonlinear Analysis: Real World Applications, vol. 11, no. 2, pp. 705-712, 2010.

[7] K. S. Sudheer and M. Sabir, "Adaptive modified function projective synchronization between hyperchaotic Lorenz system and hyperchaotic Lu system with uncertain parameters," Physics Letters. A, vol. 373, no. 41, pp. 3743-3748, 2009.

[8] X. Wang and J. Song, "Adaptive full state hybrid projective synchronization in the unified chaotic system," Modern Physics Letters B, vol. 23, no. 15, pp. 1913-1921, 2009.

[9] L. Runzi, "Adaptive function project synchronization of Rössler hyperchaotic system with uncertain parameters," Physics Letters. A, vol. 372, no. 20, pp. 3667-3671, 2008.

[10] G. H. Erjaee and M. Alnasr, "Phase synchronization in coupled Sprott chaotic systems presented by fractional differential equations," Discrete Dynamics in Nature and Society, vol. 2009, Article ID 753746, 10 pages, 2009.

[11] N.-S. Pai and H.-T. Yau, "Robust exponential converge controller design for a unified chaotic system with structured uncertainties via LMI," Discrete Dynamics in Nature and Society, vol. 2010, Article ID 948590, 10 pages, 2010.

[12] H.-T. Yau and C.-L. Chen, "Chaos control of Lorenz systems using adaptive controller with input saturation," Chaos, Solitons and Fractals, vol. 34, no. 5, pp. 1567-1574, 2007.

[13] H.-T. Yau, J.-S. Lin, and J.-J. Yan, "Synchronization control for a class of chaotic systems with uncertainties," International Journal of Bifurcation and Chaos in Applied Sciences and Engineering, vol. 15, no. 7, pp. 2235-2246, 2005.

[14] R. Mainieri and J. Rehacek, "Projective synchronization in three-dimensional chaotic systems," Physical Review Letters, vol. 82, no. 15, pp. 3042-3045, 1999.

[15] Y. Yu and H.-X. Li, "Adaptive generalized function projective synchronization of uncertain chaotic systems," Nonlinear Analysis: Real World Applications, vol. 11, no. 4, pp. 2456-2464, 2010.

[16] Y. Chen, H. An, and Z. Li, "The function cascade synchronization approach with uncertain parameters or not for hyperchaotic systems," Applied Mathematics and Computation, vol. 197, no. 1, pp. 96-110, 2008. 
[17] H. An and Y. Chen, "The function cascade synchronization method and applications," Communications in Nonlinear Science and Numerical Simulation, vol. 13, no. 10, pp. 2246-2255, 2008.

[18] D. Matignon, "Stability results of fractional differential equations with applications to control processing," in Proceedings of the IMACS/IEEE SMC Multiconference on Computational Engineering in Systems Applications, pp. 963-968, Lille, France, 1996.

[19] C. Li and G. Peng, "Chaos in Chen's system with a fractional order," Chaos, Solitons and Fractals, vol. 22, no. 2, pp. 443-450, 2004.

[20] I. Grigorenko and E. Grigorenko, "Chaotic dynamics of the fractional Lorenz system," Physical Review Letters, vol. 91, no. 3, Article ID 034101, 4 pages, 2003.

[21] G. Chen and T. Ueta, "Yet another chaotic attractor," International Journal of Bifurcation and Chaos in Applied Sciences and Engineering, vol. 9, no. 7, pp. 1465-1466, 1999.

[22] M.S. Tavazoei and M. Haeri, "A necessary condition for double scroll attractor existence in fractionalorder systems," Physics Letters A, vol. 367, no. 1-2, pp. 102-113, 2007. 


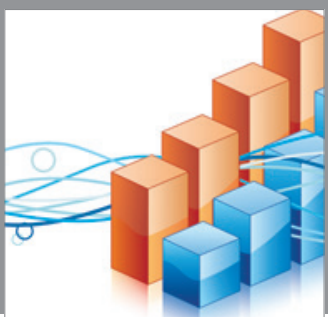

Advances in

Operations Research

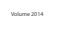

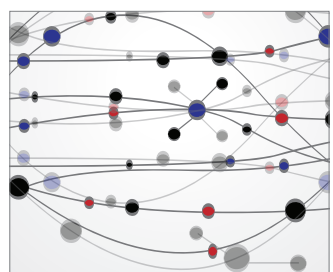

\section{The Scientific} World Journal
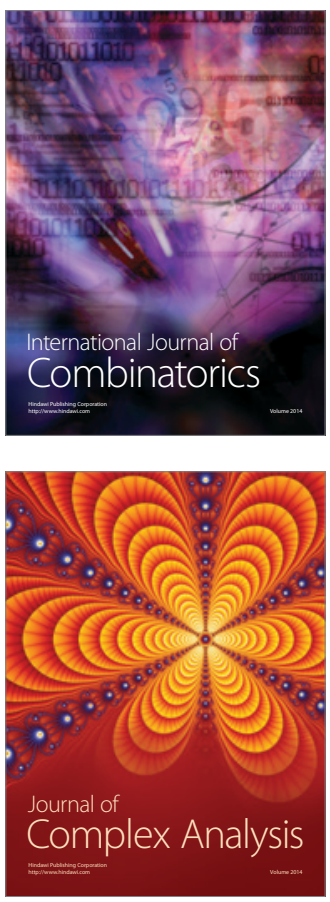

International Journal of

Mathematics and

Mathematical

Sciences
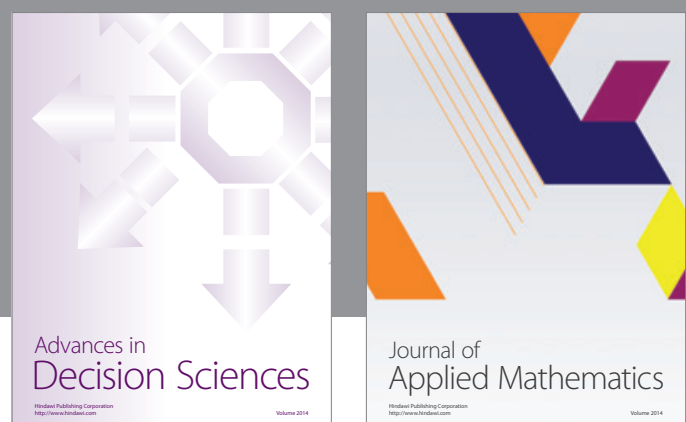

Journal of

Applied Mathematics
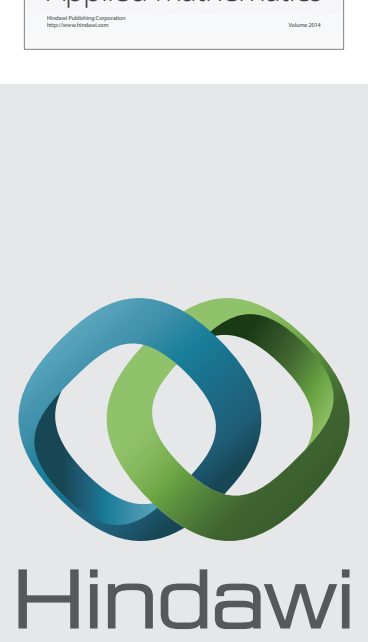

Submit your manuscripts at http://www.hindawi.com
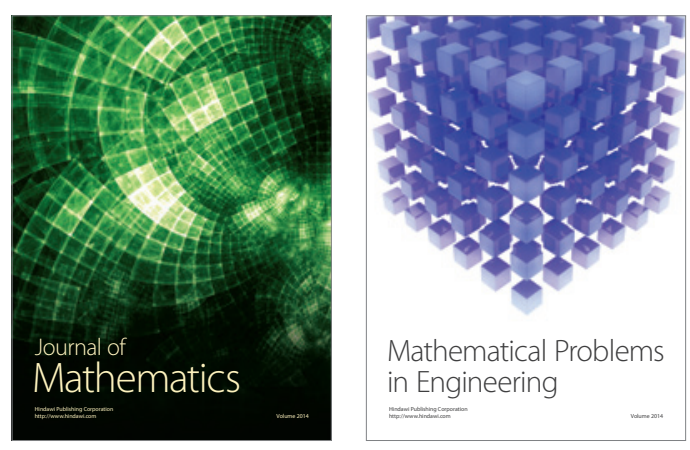

Mathematical Problems in Engineering
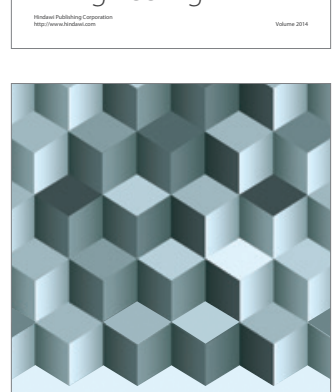

Journal of

Function Spaces
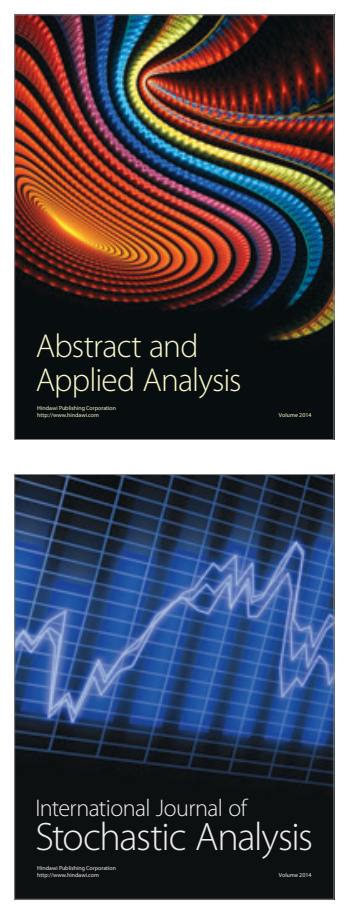

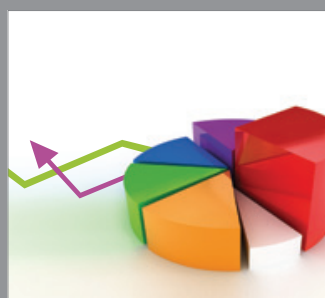

ournal of

Probability and Statistics

Promensencen
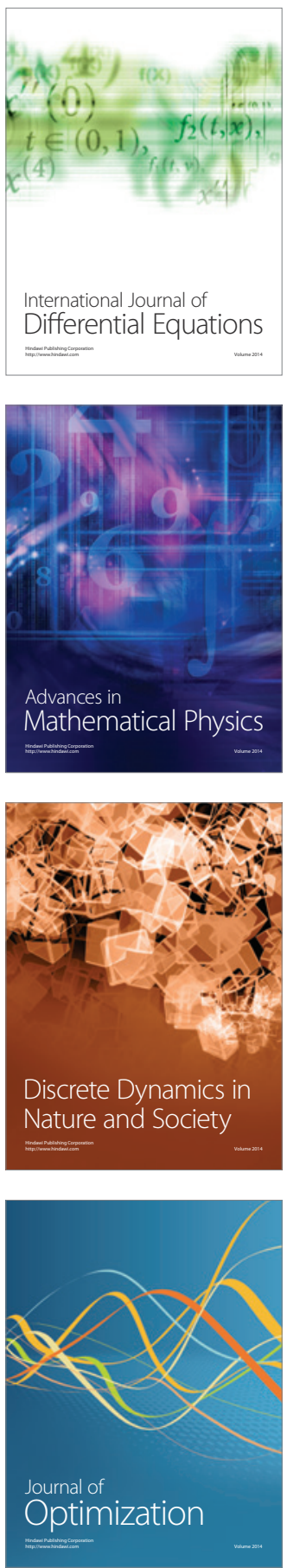